\section{STAINLESS STEEL NORMALIZED POLARIZATION CURVES}

M.G.Sustersic*, L.H.Pesetti, C.Abaca, T.A. Zanon and C. Grzona

Facultad de Ingenieria y Ciencias Económico-Sociales. Universidad Nacional de San Luis Avda. 25 de Mayo 384. C.P. 5730 Villa Mercedes (San Luis) .ARGENTINA e-mail:masus@fices.unsl.edu.ar

\section{ABSTRACT}

A technique to obtain normalized current was conceived for the cases in which the electrode surface corrodes. Each polarization curve point is obtained with an untreated electrode, which is submitted to two potential jumps: the first jump was applied to measure the current-potential relation. The second one was used for area normalization. Stainless steels 316 and $316 \mathrm{~L}$ have been used in a concentrated chloride solution.

Key Words: corrosion - normalized current - variable area - stainless steel

\section{INTRODUCCION}

If an active metal is submitted to an aggressive environment it will corrode spontaneously. Moreover, applying an anodic potential may accelerate its corrosion. In both cases the surface area will change. The objective of the present paper is to describe a technique to take into account surface area changes on corroding metals.

Experimental methods to determine real surface area have been reviewed and their limitations discussed [1]. The meaning of real surface area concept has also been explained: "The meaning of real surface area depends on the method of its measurement, on the theory of this method, and on the conditions of application of the methods. Thus, for a given system, various real surface areas can be defined, depending on the characteristic dimension of the probe used. The most appropriate real surface area is the one estimated using a method which best approaches the experimental situation to which that area is to be applied " [1].

In this sense, methods to determine real surface area in situ are better than ex situ ones. We are dealing with an electrode / electrolyte system in which the electrode is attacked by its environment. Moreover, an external potential strongly contributes to the material damage. If this polarization is stopped, the real surface area determination must be quick enough to permit the area measure before the spontaneous dissolution causes a later area change.

Therefore, the described methods for solid electrodes real area determination are not. applicable to corroding electrodes [2-18].
Techniques based on the capacitance method in two different versions: the capacitance ratio [2-5] and the Parsons Zobel plot are not adequate for our system [6]. The first may be adequate because it is a single point method and hence involves little time. On the other hand, its experimental applicability is doubtful [1]

The multiple point method [6] has been discarded because it needs much time

Hydrogen or oxygen monolayer [8-12] adsorption is not electrochemically observed on active metals. Thus, the techniques based on monolayer charge measure are not adequate.

Underpotential deposition of metals suffers from the same shortcomings as that of hydrogen adsorption method [14].

The other in situ techniques described have been discarded because they need too much time [15-18].

Furthermore, any intent to measure in situ real area of a corroding electrode must be almost impossible.

In spite of this, the normalization of each current value of a polarization curve to a selected standard value has been tried.

To determine each current / potential value of a polarization curve, the experiment starts with an untreated electrode which is subjected to a potential jump ranging from $0.0 \mathrm{~V}$ to a selected potential E. The current response is read after 5,7 or 10 minutes depending on the experiment. After current reading, the circuit is opened and the electrode is subjected to a low potential $\mathrm{E}_{0}$ during $40 \mathrm{~s}$.

$E_{0}$ is selected from the potential range at which electrode does not react and no passive film is formed on the surface. If an oxide film during the anodic polarization $\mathrm{E}$ was covered the electrode, its stay at low potential $\mathrm{E}_{0}$ allows surface film to destroy. In addition, solution stirring helps film dissolution and to remove corrosion products away from the surface. Then, the electrode is submitted to a potential jump between $\mathrm{E}_{0}$ and $\mathrm{E}_{\mathrm{f}}$. The initial current response of this final potential jump, $\mathrm{I}_{0}$ is used to normalize current I of the polarization experiment

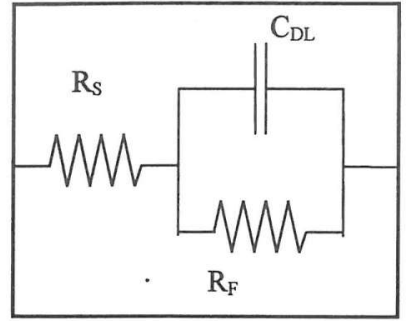

Fig. 1 - Equivalent circuit of the electrode/electrolyte interface. $C_{D L}$ double layer capacity. $\mathrm{R}_{\mathrm{S}}$ : solution resistance. $\mathrm{R}_{\mathrm{F}}$ : Charge transfer reaction. 
The current response first shows the capacitive current due to the double layer charge The equivalent circuit consists of a capacity condenser $C_{D L}$ connected in parallel with resistance, $R_{F}$. This device is connected in series with a resistance $R_{S}$. $R_{S}$ and $R_{F}$ simulate the solution resistance and the charge transfer resistance respectively

When a potential difference $E_{f}$ is applied to this circuit, the condenser is first charged through the resistance connected with it in series. This resistance consists of electrolyte solution, Rs.

A condenser charge current varies with time as follows:

$$
I=\frac{E_{f}}{R_{s}} \cdot e^{\frac{-t}{R_{S} C_{D L}}}=I_{0} \cdot e^{\frac{-t}{R_{S} C_{D L}}}
$$

Where $I_{0}$, the initial current at $t=0$, is the same as if the circuit would only have resistance $R_{S}$. Resistance depends on the length and section through resistivity $\rho$ or its inverse, the conductivity $\mathrm{\kappa}$ :

$$
R=\frac{1}{K} \frac{L}{A}
$$

In this case, $\mathrm{K}$ is the electrolyte conductivity and $\mathrm{A}$, the electrode area. As the electrode is always placed in the same position, $L / \kappa$ does not vary through the different experiments. The equation (2) leads to a direct relation between initial current and the electrode area:

$$
I_{0}=E_{f} \cdot \kappa \cdot \frac{A}{L}
$$

Thus, we have a relation to normalize the measured current value in each polarization curve point in relation to a reference area. This is performed by measuring the current jump height $\left[I_{0}\right]$, when the final potential pulse is applied. $V_{0}$ and $V_{f}$ of the second jump are equal in every point of the polarization curve obtained.

The resistance $\mathrm{R}$ in equation (2) depends only on the electrolyte resistance and on the electrode area. The electrolyte is concentrated enough to avoid any effect of the diffuse double layer.
Moreover, we suppose that reaction products do not influence electrolyte conductivity. This is nearly true if product concentration is negligible compared with the electrolyte concentration. Thus, the electrolyte solution has been changed after each experiment to avoid the contamination of the electrolyte with the corrosion products.

\section{EXPERIMENTAL}

A three compartment pyrex glass cell was used. The auxiliary electrode was a platinum sheet of about $4 \mathrm{~cm}^{2}$ separated from the working electrode by a porous glass-plate

A silver / silver chloride / chloride electrode was used as reference electrode.

The working electrodes were stainless steels AISI 316 and $316 \mathrm{~L}$ sheets. The different electrodes were of the same piece of metal. The exposed surface area in each electrode was delimited with paraffin.

The supporting electrolyte was a $1 \mathrm{M} \mathrm{KCl}$ solution prepared with analytical reagent and bidistilled water

The cell was thermostatized at $25^{\circ} \mathrm{C}$

Each polarization curve point was obtained with an untreated electrode, which was placed in the oxygen free solution and submitted to a potential jump from $0.0 \mathrm{~V}$ to a given potential. This was performed with a square wave function generator connected with a potentiostat. After 5,7 or 10 minutes (depending on the experience), the current response was read. Then, the circuit was opened and the electrode subjected to a $V_{0}=-0.3 \mathrm{~V}$ during 40 secs.

After this, a second potential jump was applied between -0.3 to $0.0 \mathrm{~V}$. Then the current response to this jump was read in a digital oscilloscope.

The initial current response $\mathrm{I}_{0}$, was used to normalize the previously obtained current value in the polarization experiment to relate it with the reference area.

Five values of normalized current were obtained for each potential. The average currents were used to construct polarization curves shown in Figs. 2 - 6 .

The electronic device consisted of a GE - M4 LYP square wave generator connected to a M5 LYP potentiostat. A Tektronic digital oscilloscope has been used to measure electrode current responses.

It must be added that reproducible normalized current could not be obtained with a potentiostat which sents current pulses to the cell when the circuit is closed. Moreover, the potential jump must be a perfect square-shape 


\section{RESULTS AND DISCUSSION}

Tables 1 and 2 show the normalized and non-normalized currents obtained with 316 stainless steel for different apparent areas and two potentials.

The normalized currents are almost constant and independent of the apparent area [Tables 1- 2]

The polarization curves obtained with AISI 316 and AISI 316 L stainless steels are shown in Figures 2-6.

To obtain each current-potential value we followed two different ways: the classical and the normalized one here dealt with. In the first way, the same electrode was used to construct the whole polarization curve. The current has been measured at a fixed time after applying the given potential. Thus, the current was read five, seven or ten minutes after applying the potential. (Figs. 2a-6a). In the second way, the procedure described above was used (Figs. 2b6b).

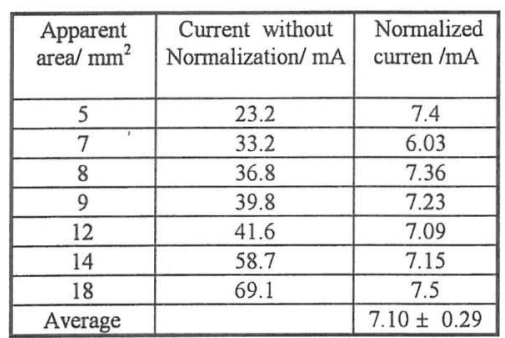

Table 1. Normalized and non normalized currents for different apparent areas electrodes of AISI 316 stainless steel. $\mathrm{E}=1.0 \mathrm{~V} ; \mathrm{t}=5 \mathrm{~min} ; \mathrm{T}=25^{\circ} \mathrm{C}$

\begin{tabular}{|c|c|c|}
\hline $\begin{array}{c}\text { Apparent } \\
\text { Area/mm }\end{array}$ & $\begin{array}{c}\text { Current without } \\
\text { normalization/mA }\end{array}$ & $\begin{array}{c}\text { Normalized } \\
\text { current/mA }\end{array}$ \\
\hline 4 & 14.08 & 4.26 \\
\hline 8 & 19.82 & 4.95 \\
\hline 14 & 31.7 & 4.17 \\
\hline 15 & 34.7 & 4.23 \\
\hline 18 & 37.6 & 4.31 \\
\hline 21 & 42.1 & 4.57 \\
\hline Average & & $4.41 \pm 0.33$ \\
\hline
\end{tabular}

Table 2.Normalized and non normalized currents for different apparent areas electrodes of AISI 316 stainless steel. $\mathrm{E}=0,60 \mathrm{~V} ; \mathrm{t}=5 \mathrm{~min} ; \mathrm{T}=25^{\circ} \mathrm{C}$
The apparent areas for the both types of experiments were the same. The differences between the curves are due to the method used to obtain them. Currents of normalized polarization curves are significantly lower than those obtained without normalization.

The main difference between the curves obtained with the two different methods is that normalized curves show a current drop due to metal oxide formation (Figs. 2b-6b). In the polarization curves obtained with the traditional method (Figs. 2a-6a), each point involves the change that the electrode suffered during the previous treatment. The area increase is so great that current lowering due to the metal passivation cannot be seen

If we use a microscope to observe an electrode treated with the old method, we see a strongly damaged metal. The metal damage is so great that pits cannot be observed.

With the new method, the experiment to obtain each polarization curve point begins with a new surface. In this case the microscope image shows only the metal attack that took place at the potential under study during the treatment at one potential only. If we observe by microscope the electrodes treated above $1,2 \mathrm{~V}$, they present clearly defined pits. Pits appear on a surface that has been previously recovered by passive films. The current drops due to surface film appear above $1.2 \mathrm{~V}$ in all the figures shown

With the classical method, one point of the polarization curve is not comparable with each other because the electrode area is not the same. The second point is obtained with a wider surface area than that of the first point. The third one is obtained with wider surface area than that of the second point and so on.

In Fig. 6 , curves $6 a, 6 a^{\prime}, 6 b$ and $6 b^{\prime}$ were obtained with the same apparent electrode areas.

In Fig. 6a, a polarization curve without normalization is shown. The Fig. $6 \mathrm{~b}$ was obtained with the normalization procedure. Lower currents than those of fig. 6a appeared. In addition, a current drop due to surface film formation is observed. The Fig. 6b' shows a polarization curves obtained with normalization and with the hydroquinone inhibitor addition [19]. As the currents in Fig. 6b' are lower than those of Fig. 6b it can be concluded that hydroquinone behaves as an inhibitor. The same conclusion cannot be taken out by comparing Figs. $6 a^{\prime}$ and $6 a$. In this case the inhibition effect is not clear because no definite current lowering is observed. In the experiment of figure $6 a$ and $6 a^{\prime}$ it has been started with the same geometric area. The geometric area has no exact meaning and the results obtained by comparing electrodes with same geometric area are not conclusive. Only through the normalization procedure, the results can be compared, to obtain reliable conclutions 


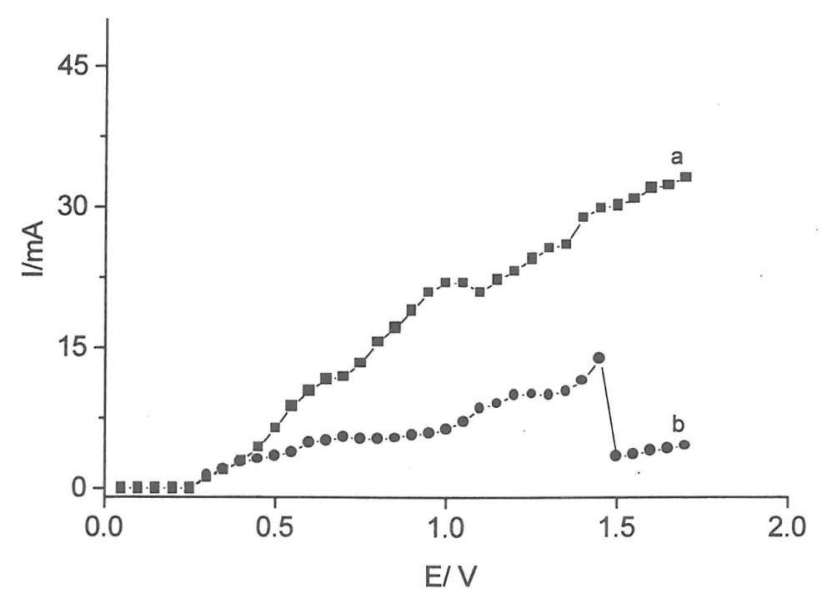

Fig 3 -Polarization curves for AISI - 316L stainless steel without normalization (a) and with normalization (b). The apparent area was the same for both experiments The currents were read after $7 \mathrm{~min}$ of potential applying. $\mathrm{T}=25^{\circ} \mathrm{C}$.

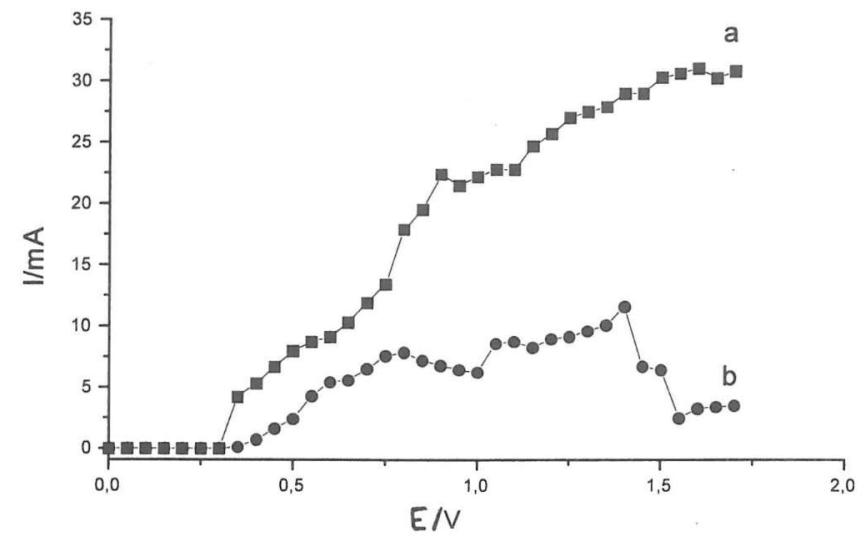

Fig. 4 - Polarization curves for AISI 316 stainless steel without normalization (a) and with normalization (b). The apparent area was the same for both experiments The currents were read after 5 min of potential applying. $\mathrm{T}=25^{\circ} \mathrm{C}$.
The current lowering due to inhibitor addition is more definite in the case of normalized currents than in the case of currents without normalization [Figs $6 a$ and $6 a^{\prime}$ ]. In this sense, the normalization procedure is useful to confirm an inhibition effect that, in other way, would be doubtful.

In all the non- normalized curves here shown [Figs 2a-6a] a change of the slope in $c a$. $0.8 \mathrm{~V}$ appears. In the corresponding normalized curves [figs $2 \mathrm{~b}-6 \mathrm{~b}$ ], a change of slope of Figs. $2 \mathrm{a}-6 \mathrm{a}$ is replaced by a current plateau between 0.6 and $1.0 \mathrm{~V}$. The first plateau and the following current drop are probably due to the different nature of the surface film formed. In the experimental condition of the figures, a solid ferric and non-stoichiometric oxides are permitted [22]

However, it must be clarified that the potentiokinetic method here used to construct the polarization curves [20] cannot lead to definitive conclutions about the stationary state of the system. Moreover, it is not possible to conclude that the observed current drops are due to a true passivating film, because the currents after the current drops are too high to be called passive currents and in addition they continue increasing.

With corroding electrodes, stationary currents are very difficult to obtain. A current that seems stationary may be found. After ten minutes it changes again and so on. As the metal corrodes the area changes. As the are changes, current changes, and so on.

To compare two experimental results obtained with two different pieces of the same metal they must be treated in the same manner. The way to apply the potential must be the same Here, a square step is used and the current reading time after the potential applying is also fixed.

The normalization procedure does not lead to current drop appearance in all the cases. In stainless steels 304 and 304 L studies, only a current lowering [21] through the normalization procedure here described is observed. 


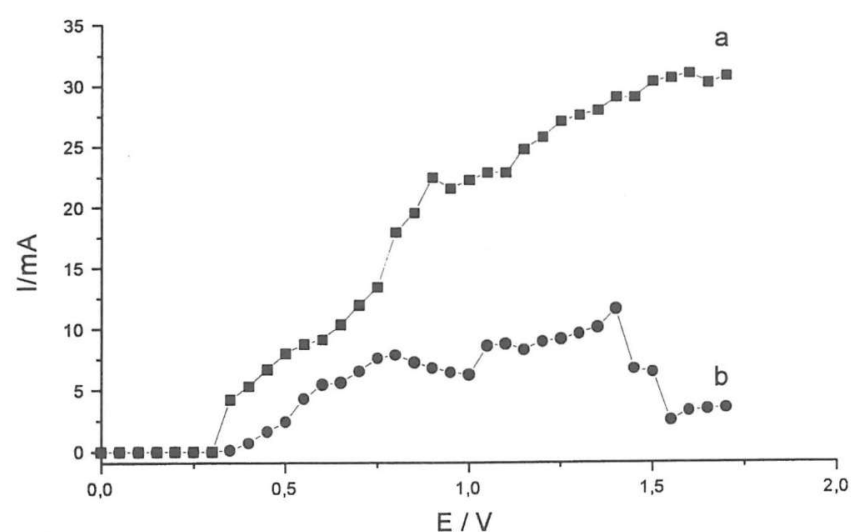

Fig. 4 - Polarization curves for AISI 316 stainless steel without normalization (a) and with normalization (b). The apparent area was the same for both experiments. The currents were read after $5 \mathrm{~min}$ of potential applying. $\mathrm{T}=25^{\circ} \mathrm{C}$.

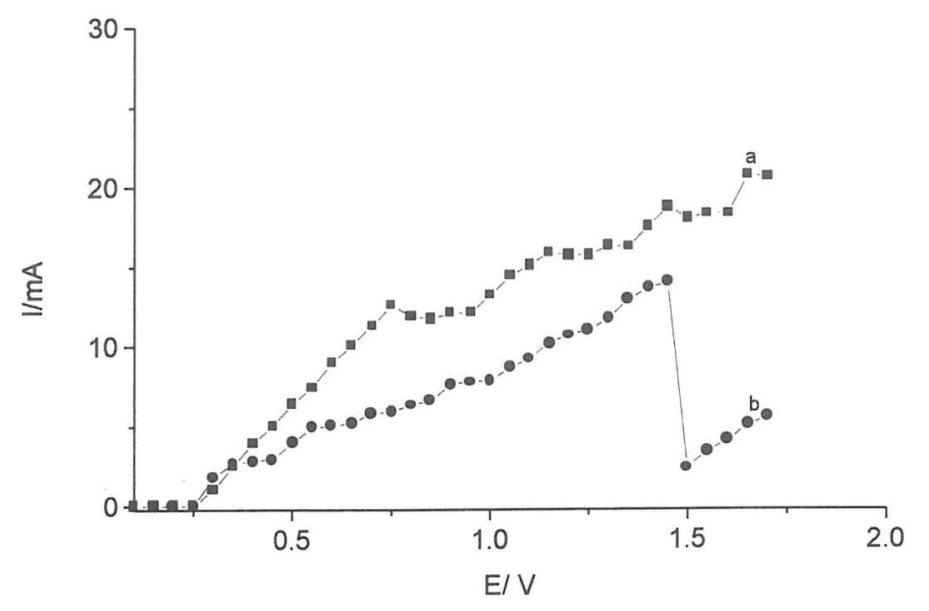

Fig 5 - Polarization curves for AISI- 316 stainless steel without normalization (a) and with normalization (b). The apparent area was the same for experiments. The currents were read after $7 \mathrm{~min}$ of potential applying. $\mathrm{T}=25^{\circ} \mathrm{C}$

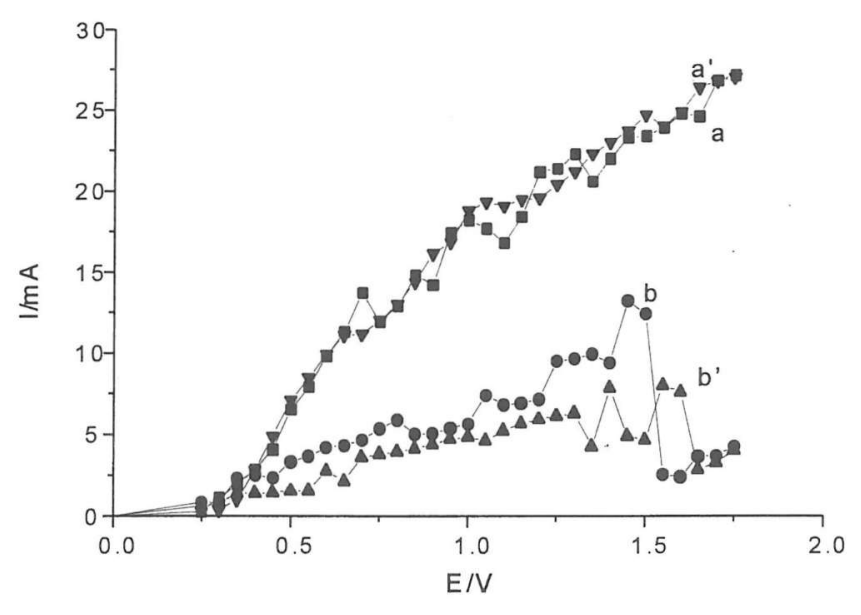

Fig.6 - Polarization curves for AISI - 316 stainless steel without normalization (a) and with normalization (b) ; (a') and (b') were obtained in the same conditions as (a) and (b) with $5 \times 10^{-3}$ hydroquinone inhibitor addition. The currents were read after 10 min of potential applying. $\mathrm{T}=25^{\circ} \mathrm{C}$

\section{CONCLUSIONS}

The current values of a polarization curve obtained with an electrode, whose area changes, must be normalized to take account the area changes. A pulse technique to do this is here proposed.

With this technique, polarization curves with lower currents than those of a nonnormalized polarization curves were obtained with 316 stainless steel electrodes. At the same time, current drops due to surface film formation were found at experimental conditions at which ordinarily are not observed

With the new technique an inhibition effect of hydroquinone addition is clearly observed.

\section{ACKNOWLEDGMENTS}

This investigation was supported by a grant (P-519001) awarded by the Universidad Nacional de San Luis and by the Secretaría de Ciencia y Técnica. We acknowledge to Dr. Arvía his valuables observations 


\section{REFERENCES}

1- S.Trasatti and O.A.Petrii. Electroanal. Chem. $\underline{327}$ (1992) 353-376.

2- M Breiter, J.Electroanal. Chem. 81 (1997) 275.

3- J. M. Novoselkii, N. I. Konevskikh, L. Ya. Egorov and E. P. Sidorov, Elektrokhimiya $\underline{7}$ (1971) 393.

4- E. Robert, Oberfl.-Surf. 22 (1981) 261. T. Ohmori, J. Electroanal. Chem. 157 (1983) 159.

5- T. Ohmori, J. Electroanal. Chem. 157 (1983) 159.

6- R. Parsons and F. R.G. Zobel, J. Electroanal. Chem 9, (1965) 333.

7- F.G. Will and C. A. Knorr, Z. Elektrochem. 64 (1960) 258

8- S. Gilman, J Phys. Chem. 67 (1963) 78, J. Electroanal. Chem. 7 (1964) 382.

9- S. Gilman, J.. Phys. Chem. 71 (1967) 4339.

10- R. Woods. J. Electroanal. Chem. 49 (1974) 217.

11- D.V. Sokolskii, B. Yu, Nogorbekov, N. N. Gudeva and R. G. Mustafina, Elektrokhimiya 22 (1986) 1185.

12- A.G. Pshenichnikov, Mat. Chem. Phys. 22 (1989) 121.

13- M. Breiter, K. Hoffmann and C. Knorr, Z. Elektrochem. 61 (1957) 1168.

14- T. D. Gladisheva, B. J. Podlovchenko and Z. A. Zikrina, Elektrokhimiya 23 (1987) 1446

15- G. Singh, M. H. Miles and S. Srinivasan in A.D. Franklin (Ed.), Electrocatalysis on Non Metallic Surfaces, NBS Spec. Publ. N. 455 (1976) 289.

16- J. O’M. Bockris and T. Otagawa, J. Electrochem. Soc. 131 (1984) 290.

17- L. D. Burke and O. J. Murphy, J. Electroanal. Chem. 96 (1979) 19.

18- C. H. Giles and A. P. D’Silva, Trans. Faraday Soc. 65 (1969) 1943, 2516.

19- C. Grzona, N. Almeida and M.G. Sustersic (in preparation).

20- J.R. Galvele, Cursos Ecomar; Ecomar /006/A 1975.

21- L Pesetti, C. Abaca and M.G.Sustersic (in preparation).

22- M. Pourbaix, "Atlas of Electrochem. Equilibria." Pergamon Press, Cebelcor, Bruselas (1966). 